\title{
Las mujeres en Lefebvre: por una relectura del espacio urbano desde los feminismos marxistas
}

\author{
Women on Lefebvre: Towards a re-reading of urban \\ space from Marxists Feminisms
}

\author{
As mulheres em Lefebvre: para uma releitura do \\ espaço urbano a partir dos feminismos marxistas
}

\section{Almendra Aladro ${ }^{\circ}$}

Resumen Este artículo propone revisitar el espacio social conceptualizado en la obra del sociólogo francés Henri Lefebvre desde una visión unitaria de la explotación capitalista y la opresión de la mujer. Para ello, se realiza una revisión bibliográfica para tender puentes entre una de las nociones más significativas de las luchas por los derechos económicos, sociales y culturales en América Latina y una teoría que, habiendo iniciado sus pasos tímidamente hacia finales de la década del 7o, renace en la actualidad frente a propuestas teóricas que excluyen de sus análisis de género la cuestión de clase. Se discurre a través del obrerismo de Federici para rescatar sus aportes en relación a la conformación de las ciudades durante la transición capitalista y el proceso de expulsión y pauperización social de las mujeres, mientras que se prefiere la Teoría de la Reproducción Social para lograr un marco analítico que permita dar cuenta del fenómeno urbano contemporáneo y el lugar de las mujeres en él. Se concluye que el razonamiento de Lefebvre, además de caer en preconceptos típicos de su época en relación a las reivindicaciones de las mujeres, utiliza un concepto muy amplio de reproducción social que lo hace perder de vista su importancia para el capital en igualdad con la producción de mercancías. La complementación de la teoría lefebvriana con los aportes de los feminismos marxistas, entonces, facilitan el entendimiento del lugar histórico de las mujeres como primeras desposeídas de lo urbano y su rol actual en el despliegue de estrategias colectivas de reproducción social que significan una apropiación del espacio abstracto que le gana terreno tanto a su pérdida histórica de status como a la lógica contemporánea del capital. Palabras Clave Feminismo. Marxismo. Reproducción Social. Ciudad. Espacio Urbano.

a Abogada (UNMDP). Candidata al título de Doctora en Derecho (UNMDP) Becaria doctoral (CONICET) con lugar de trabajo en el Centro de Investigación y Docencia en Derechos Humanos “Dra. Alicia Moreau” (CIC-CIDDH-UNMDP). Correo electrónico: aaladro@mdp. edu.ar 
Abstract This article proposes to revisit the social space conceptualized in the work of the French sociologist Henri Lefebvre from a unitary vision of capitalist exploitation and the oppression of women. For this, a bibliographic review is carried out to build bridges between one of the most significant notions of the struggles for economic, social and cultural rights in Latin America and a theory that, having timidly started its steps towards the end of the 7os, reborns nowadays in the face of theoretical proposals that exclude class from gender analysis.

It runs through Federici's workerism to rescue her contributions in relation to the conformation of cities during the capitalist transition and the process of expulsion and social impoverishment of women, while the Theory of Social Reproduction is preferred to achieve an analytical framework that allows to account for the contemporary urban phenomenon and the place of women in it.

It is concluded that Lefebvre's reasoning, in addition to falling into typical preconceptions of his time in relation to the demands of women, uses a very broad concept of social reproduction that makes him lose sight of its importance for capital in equality with production of goods. The complementation of the Lefebvrian theory with the contributions of Marxist Feminisms, then, facilitates the understanding of the historical place of women as the first dispossessed of the urban and their current role in the deployment of collective strategies of social reproduction that mean an appropriation of abstract space that gains ground both from its historical loss of status and from the contemporary logic of capital. Keywords Feminism. Marxism. Social Reproduction. City. Urban Space.

Resumo Este artigo se propõe a revisitar o espaço social conceituado na obra do sociólogo francês Henri Lefebvre a partir de uma visão unitária da exploração capitalista e da opressão das mulheres. Para isso, é realizada uma revisão bibliográfica que visa construir pontes entre uma das mais significativas noções das lutas pelos direitos econômicos, sociais e culturais na América Latina e uma teoria que, tendo iniciado timidamente seus passos no final dos anos 70, renasce hoje diante de propostas teóricas que excluem a questão de classe de sua análise de gênero.

Percorre o obreirismo de Federici para resgatar suas contribuições em relação à conformação das cidades durante a transição capitalista e o processo de expulsão e empobrecimento social das mulheres, enquanto a Teoria da Reprodução Social é preferida para alcançar um arcabouço analítico que permita dar conta do fenômeno urbano contemporâneo e o lugar da mulher nele.

Conclui-se que o raciocínio de Lefebvre, além de cair em preconceitos típicos de sua época em relação às demandas das mulheres, utiliza um conceito muito amplo de reprodução social que o faz perder de vista sua importância para o capital em igualdade com a 
produção de bens. A complementação da teoria lefebvriana com as contribuições dos feminismos marxistas, então, facilita a compreensão do lugar histórico das mulheres como primeiras despossuídas do urbano e seu papel atual no desdobramento de estratégias coletivas de reprodução social que significam uma apropriação do espaço. Resumo que ganha terreno tanto com sua perda histórica de status quanto com a lógica contemporânea do capital.

Palavras-chave Feminismo. Marxismo. Reprodução social. Cidade. Espaço Urbano.

\section{INTRODUCCIÓN}

La afirmación de que la ciudad es un producto histórico de las relaciones de dominación data de unas cuantas décadas de antigüedad. A partir del desarrollo de la idea de derecho a la ciudad de Henri Lefebvre en 1969 como denuncia o exigencia de la clase obrera, estudiar la "cuestión urbana" es reflejar tensiones socio-espacio-temporales y, muy especialmente, el estado de la lucha de clases frente a un grado histórico de desarrollo de las fuerzas productivas en un territorio determinado. Desde el punto de vista jurídico, el derecho a la ciudad pierde su origen de lucha y pasa a vincularse "con lo que el Estado está dispuesto a ceder para la gestión de la ciudad, teniendo así un entendimiento limitado del concepto" (Canestraro, 2016, p. 60) para quedar enmarcado dentro de los derechos humanos "emergentes" y se lo ha emparentado con los derechos económicos, sociales, culturales y ambientales (en adelante, DESCA). En América Latina, su reconocimiento fue liderado por la Constitución Federal de Brasil y, en Argentina, es reconocido como principio rector de la Ley de Acceso Justo al Hábitat no 14449 de la provincia de Buenos Aires y limitado en su contenido.

La exclusión de la especificidad de las mujeres en los entornos urbanos llevó a la elaboración de críticas a esta conceptualización inicial del derecho a la ciudad desde los estudios de género en los campos de la geografía, el urbanismo, la sociología y la antropología principalmente. En Argentina y a nivel regional, Ana Falú es un referente indiscutible que ha puesto en agenda la violencia de género en las ciudades latinoamericanas tomando como punto de partida ideas de Jane Jacobs y Doreen Massey principalmente. Así, da cuenta de la invisibilidad de las mujeres en las ciudades y de cómo los usos de los bienes urbanos tienen correlatividad con la división sexual del trabajo. La autora también enseña que las ciudades "se perciben de manera diferencial según los sujetos sociales" (Falú, 2014, p. 15) y ello "se encuentra estrechamente relacionado a los modos de acceso, a las condiciones y calidades de vida que la ciudad les ofrece", es decir, "quiénes y cómo usan y disfrutan el conjunto de bienes urbanos que refieren a derechos ciudadanos" (Falú, 
2014, p. 15). Plantea, en este sentido una agenda para la sensibilización y el avance en el derecho al uso y disfrute de las ciudades desde una perspectiva teórica que prioriza la opresión sobre la explotación. Las elaboraciones teóricas enroladas en esta línea toman a las mujeres como grupo y, a partir del estudio de su relación con la ciudad y los bienes urbanos, realizan propuestas de reforma para la mejora de la calidad de vida urbana desde una perspectiva de género más hermanada a los desarrollos vinculados a teorías decoloniales.

Otras líneas de trabajo a nivel regional, como las planteadas por Paola Jirón y Paula Soto Villagrán, ponen el eje del análisis en las estrategias de la movilidad de las mujeres para erguir sus críticas a la planificación de las ciudades capitalistas, que deprecia la importancia de los desplazamientos de las mismas principalmente vinculados a las tareas de cuidado. Este tipo de aportes vienen de la mano de las propuestas de "ciudades cuidadoras", es decir, espacios urbanos planificados no sólo desde la producción de mercancías sino también de las tareas históricamente relegadas a las mujeres. En este sentido, este artículo viene a dar una mirada de clase que tiene como pretensión superar la idea de "cuidado" - familiar, privado, individual - trayendo la Teoría de la Reproducción Social para complejizar el debate desde el clasismo.

Las investigaciones sobre el uso diferencial de los bienes urbanos y la configuración de prácticas urbanas propias - tanto con el foco puesto en la violencia de género como en las tareas de cuidado - han llevado a un distanciamiento de las ideas iniciales de Lefebvre con respecto a la ciudad, incluso rompiendo con algunos de los presupuestos básicos de sus planteos para dar lugar a nuevas teorizaciones alejadas de la tradición marxista. Precisamente, aquí se busca realizar un aporte dentro del paradigma lefebvriano desde la histórica problematización alrededor de la clase y el género (Skeggs, 2019).

Este artículo propone revisitar el espacio social conceptualizado en la obra del sociólogo francés Henri Lefebvre desde una visión unitaria de la explotación capitalista y la opresión de la mujer. Para ello, se realiza una revisión bibliográfica para tender puentes entre una de las nociones más significativas de las luchas por los derechos económicos, sociales, culturales y ambientales en América Latina y una teoría que, habiendo iniciado sus pasos tímidamente hacia finales de la década del 70, renace en la actualidad frente a propuestas teóricas que excluyen de sus análisis de género la cuestión de clase.

El escrito parte de dos preconceptos. El primero es que en los estudios actuales sobre el derecho a la ciudad existe sectorialidad en cuanto a las obras que abordan el lugar de la mujer en la urbe contemporánea y que esta sectorialidad se caracte- 
riza por la exclusión del estudio de la explotación capitalista (clase) en favor de la problematización de la opresión de la mujer (género), generando incluso aportes desde tradiciones teóricas alejadas del marxismo. El segundo es que existe un sesgo de género respecto de las dimensiones que componen al derecho a la ciudad como capacidad explicativa y otro sesgo en las políticas de aplicación concreta de este derecho aún en su mejor versión posible.

La estructura del artículo reconoce cuatro partes claramente diferenciadas. La primera está destinada a sintetizar el lugar de las mujeres y de la reproducción social en la obra de Henri Lefebvre. Seguidamente, se busca resumir teóricamente el lugar de las mujeres en las ciudades preindustriales y su evolución conforme el desarrollo de las fuerzas productivas, para lo cual se genera un diálogo entre los escritos de Silvia Federici y las investigaciones sobre ciudades coloniales y políticas urbanas en el Virreinato del Río de la Plata. Luego, se rastrean los aportes más significativos al estudio de las ciudades traídos por la Teoría de la Reproducción Social, resaltando especialmente la complejización de la interpretación del lugar de las mujeres en las mismas desde este perspectiva. Finalmente, se presentan las conclusiones, buscando plantear nuevos puntos de partida para continuar la discusión académica.

\section{LA REPRODUCCIÓN SOCIAL Y LAS MUJERES EN LA OBRA DE HENRI LEFEBVRE}

En la noción lefebvriana de espacio social conviven en una totalidad abierta las dimensiones mental, física y social, interactuando en unidad dialéctica. Los tres elementos que componen esa tríada son a) las prácticas espaciales - espacio percibido - , b) las representaciones del espacio - espacio concebido - , y c) los espacios de representación - espacio vivido - (Lefebvre, 2013 [1974]). Mientras que las primeras son aquellas que hacen a la reproducción de la totalidad de la vida social y las segundas a las formas de conocimiento abstracto conectadas con los aparatos de poder formales e institucionales que gobiernan el espacio, los terceros se asocian directamente a la experiencia vivida desde la práctica creativa y la resistencia social (Butler, 2012). El entendimiento de cómo lo mental, lo físico y lo social se relacionan entre sí debería impedir que, dentro de este paradigma, se parcelen las investigaciones en detrimento de una o varias partes del espacio social. No obstante esto, una pesquisa rápida de las investigaciones desarrolladas tras la recuperación de la obra de Lefebvre en el último tiempo da cuenta de cómo se han descuidado los estudios sobre la reproducción social en relación a aquellos que indagan sobre mercado inmobiliario, políticas de vivienda social, instrumentos 
de planificación territorial, conflictos urbanos desde la perspectiva del acceso al suelo, entre otros tópicos.

Lefebvre (2013 [1974]) indica al espacio social como el conjunto de las relaciones de producción como las de reproducción, siendo estas últimas representadas simbólicamente mediante símbolos sexuales de lo femenino y lo masculino que, a su vez, son acompañados por otros como los de la edad. Esa simbolización divide las relaciones en codificadas y ocultas (Lefebvre, 2013 [1974]). Las relaciones sociales de reproducción - que define como "relaciones biofisiológicas entre los sexos, las edades, con la específica organización familiar" - y las relaciones de producción - dentro de las que se encuentra "la división del trabajo y su organización, por tanto (...) las funciones sociales jerarquizadas" - (Lefebvre, 2013 [1974], p. 91), se encuentran en la práctica social con "lugares específicos y conjuntos espaciales propios de cada formación social; práctica que asegura la continuidad en el seno de una relativa cohesión" (Lefebvre, 2013 [1974], p. 92).

El capitalismo logra su asentamiento a través de la escisión del valor, es decir, la separación de la producción y la reproducción, lo cual tuvo su expresión y mediación en la imbricación de los lugares destinados a: a) la reproducción biológica, b) la reproducción de la fuerza de trabajo, y c) la reproducción de las relaciones sociales de producción, identificándose respectivamente con la familia, la clase obrera y las relaciones constitutivas de la sociedad capitalista (Lefebvre, 2013 [1974]). Dentro de esa práctica ubica a la familia, la cual define como "base de las relaciones personales y directas, ligadas a la naturaleza, la tierra, a la procreación - y por tanto a la reproducción - (...) (Lefebvre, 2013 [1974], p. 94) que debe ser por ello conservada.

La escisión del valor, entonces, dio lugar al espacio abstracto, un "espacio formal y cuantitativo que niega las diferencias, tanto que proceden de la naturaleza y del tiempo (histórico) como las que vienen del cuerpo, la edad, el género y la etnia” (Lefebvre, 2013 [1974], p. 108). El derecho a la diferencia en relación dialéctica con el derecho a la ciudad - la potestad de la ciudadanía urbana a la que aspira el autor - provee las bases estratégicas para políticas de autogestión que pueden combatir las lógicas que gobiernan el espacio abstracto (Butler, 2012).

El académico francés identificó la hegemonía masculina en la producción del espacio la cual, como ya se ha dicho, responde a intereses de clase (Lefebvre, 2013 [1974]) y realiza a lo largo de su obra comentarios respecto a la situación de la mujer en el espacio y en la historia. En este sentido, autores como Chris Butler (2012) afirman que se lee en su obra un interés por la opresión en términos de pluralidad, movimientos sociales y alienación. Pese a ser contemporáneo al 
movimiento feminista materialista francés, las referencias a la mujeres y a la reproducción social repiten varias de las deficiencias endilgadas al marxismo en sus inicios - principalmente a Marx mismo. Así, aparecen reflexiones sobre cómo los electrodomésticos destinados al quehacer cotidiano aceleran el trabajo doméstico y dan tiempo libre a la mujer "para hacer tonterías o para aburrirse" (Lefebvre, 1978, p. 87) mientras que la diversidad del trabajo doméstico las libera de la carga que padecen los obreros industriales, o sobre cómo las mujeres saben clasificar a otra mujer "de una ojeada (...) más y mejor que los hombres (...) y también clasificar a los hombres en categorías convenientes" (Lefebvre, 1978, p. 95), el cortejo de "cosas" en la calle por parte de las mujeres (Lefebvre, 1978, p. 96), y su renovación en la maternidad y la infancia ante el hastío de la cotidianidad. Más polémicas son aún sus advertencias respecto a la organización de las mujeres y la posibilidad de un régimen hembrista reaccionario respecto del machismo que empapa las relaciones sociales capitalistas. No obstante ello, el autor demanda el reconocimiento del trabajo doméstico como trabajo social (Lefebvre, 1961) y denuncia el peso aún mayor que sobre las mujeres impone la cotidianidad capitalista (Lefebvre, 1978), criticando además la ilusión de que el desembarco de la mujer al trabajo industrial sería la resolución a los "viejos conflictos como se creía en un período de evolucionismo" (Lefebvre, 1978, p. 99).

En este trabajo se afirma que el sociólogo francés sólo pudo superar parcialmente los problemas que el propio Marx tuvo al momento de definir el lugar histórico de la mujer en las relaciones sociales. Como se ve en los párrafos precedentes, el autor pudo soltar la idea del trabajo industrial como solución a la “condición femenina” (como la llama en su obra De lo rural a lo urbano de 1978). Sin embargo, siguió arrastrando el carácter natural/biológico de la división sexual del trabajo, y, por lo tanto, de "lo femenino" y "lo masculino", lo cual se deriva de su asociación entre la mujer, lo cotidiano y la maternidad, y, sobre todo, de su insistencia en las "diferencias fisiológicas".

Estas deficiencias son las que lo llevan a elaborar dos tesis que falsamente le atribuye al movimiento feminista que fue a él contemporáneo. La primera, es un reduccionismo en la caracterización de la lucha de las mujeres a dos bandos: mientras que, por un lado, se le niega todo sentido a la afirmación "las mujeres" porque no constituyen un grupo social, sino que pertenecen a grupos y clases siendo las diferencias sociales de tipo biológicas y no derivadas del conocimiento "de lo real de lo humano", por lo que hablar de esa manera de las mujeres es sacarlas de la sociedad "traduciendo un prejuicio de <<los hombres $>>$ " (Lefebvre, 1978, p. 99); por el otro, se afirma la repercusión de las diferencias fisiológicas en la vida 
social que las unen contra los hombres en un grupo informal. Es decir, sea cual sea la parte del conflicto que se busque "defender", ambas tienen en común, según Lefebvre a) la definición de la mujer en función de la presencia de ciertos órganos y funciones sexuales; y b) la definición de la mujer por oposición a la del hombre.

La segunda tesis, derivada claramente de la anterior, se llama aquí "la ambigüedad de la situación de las mujeres" (Lefebvre, 1978, p. 101) y puede sintetizarse de la siguiente manera: el avance hacia un status mejorado de las mujeres puede derivar en a) una distopía matriarcal gracias a la introducción de la tecnología en la vida cotidiana y al deseo de venganza de las mujeres frente a la histórica dominación masculina (Lefebvre, 2013 [1974]; 1978); o b) la adopción de una forma ambigua "como testimonia una prensa y una literatura que se esfuerzan extrañamente en unir el sueño despierto a la práctica cotidiana” (Lefebvre, 1978, p. 100). Entonces, o bien las mujeres se apropian de las armas del amo para someterlo en las mismas condiciones que ellas fueron sometidas, o bien las mujeres quedan presas de la dominación que en realidad encubre el discurso feminista - como si, además, este último fuera exclusivamente liberal.

Al mismo tiempo, pese a su clara delimitación respecto a la importancia del estudio de la producción y la reproducción como unidad, la obra del autor conduce a una predominancia en el estudio de las relaciones de producción y esto dio lugar a que la proliferación de sus continuidades teóricas priorizaran las críticas a las ciudades-mercancía y dieran un lugar muy secundario al estudio de las infraestructuras urbanas destinadas a la reproducción de la fuerza de trabajo $y$, por ende, al rol de las mujeres en las relaciones sociales capitalistas, como se verá en los apartados siguientes. Para iniciar esa labor, a continuación se esboza un repaso teórico que busca incorporar a la opresión de género a la formación del espacio abstracto.

\section{EL LUGAR DE LAS MUJERES EN LA CIUDAD}

Al explicar la acumulación primitiva precapitalista desde una perspectiva de género, Silvia Federici $(2010 ; 2018)$ da cuenta del lugar de las mujeres en las ciudades europeas del siglo XV, momento histórico en el que se llevó adelante una contrarrevolución que buscó aplacar al proletariado consolidado y organizado tras la escasez de mano de obra producida por la peste negra en todo el continente. La política sexual desplegada incluyó el cese de la persecución de las violaciones contra las mujeres proletarias como delito y la institucionalización de la prostitución. Así, con el despojo del propio cuerpo, el territorio-cuerpo percibido como disponible en palabras de Ana Falú (2014; 2016), se buscó disciplinar a las proletarias urbanas a 
la par que se obtenía como resultado la degradación social de todas las mujeres y insensibilización de la población en general frente a la violencia contra ellas ejercida.

En la transición al capitalismo, la degradación social de las mujeres se profundizó con la escisión del trabajo reproductivo respecto de la producción de valor que, a la par de la pauperización general de la clase obrera, generó mujeres proletarias tan desposeídas como los varones proletarios, pero con cada vez menos acceso al salario y condenadas a una pobreza crónica que las convirtió en económicamente dependientes y laboralmente invisibles.

Este proceso de degradación social de las mujeres tuvo su expresión espacial, del cual Silvia Federici (2010) da cuenta con un nivel de detalle magistral. Las mujeres fueron expulsadas de la mayoría de los trabajos asalariados y también de las calles, las cuales pasaron a ser espacios peligrosos para ellas y no recomendados, al igual que los espacios públicos en general. Asimismo, se intentaba impedir que las mujeres se reunieran con sus amigas o visitaran con frecuencia a su familia de origen una vez casadas y pasaron a ser legalmente pupilas de sus esposos o padres.

Con el advenimiento de las revoluciones burguesas, lejos de lograrse la igualdad y fraternidad entre hombres y mujeres, los varones consolidaron su hegemonía y perpetuaron su dominación respecto de las mujeres delimitando con claridad, además, espacios en consecuencia. Así, el principio de reserva traído por el constitucionalismo liberal excluyó de la esfera del Estado las relaciones de carácter familiar, privadas, mientras se erigió con firmeza en el ámbito de la administración política, pública, institucionalizando, a través de las leyes nacionales derivadas de estas constituciones, la infantilización legal.

En las ciudades que luego serían parte de Argentina ${ }^{1}$ la situación de las mujeres tuvo un desarrollo similar, signado por las particularidades coyunturales coloniales y postcoloniales. Si bien la convivencia en las ciudades latinoamericanas no era uniforme, sí se ha afirmado que el uso de los espacios no estaba regido por la dictomía público-privado (Zamora, 2010). Esa diferenciación fue un proceso de largo plazo, con rupturas y continuidades, que no se agotó con delimitaciones jurídico políticas ni con la propia Revolución de Mayo. La consolidación de un poder externo - público - fue la que lentamente trasladó las facultades de control del padre de familia - privado - al Estado y espacialmente significó el traslado de la sociabilidad y la representación del poder en la imagen de la ciudad a través

1 Debo agradecer en este punto no sólo la evaluación exhaustiva desarrollada en el proceso editorial, sino también los intercambios y aportes de la querida Agustina Vaccaroni, quien en su amplia generosidad ha dedicado buena cantidad de tiempo a enriquecer este escrito desde su campo disciplinar y desde su propia investigación. 
de distintos simbolismos primero (Zamora, 2010; Favelukes, 2011) y luego con la institucionalización de figuras que inicialmente impartían justicia vecinal y también ejercían funciones de policía (Vaccaroni, 2020).

Con el crecimiento de las ciudades durante las reformas borbónicas, las familias urbanas se establecían domésticamente bajo Casas pobladas, donde multitud de sirvientes y esclavos vivían bajo la protección del hombre propietario. En general, el pater familias acumulaba su calidad de vecino por detentar la tierra, gozaba de derechos políticos y aseguraba la reproducción social en la colonia(Zamora, 2010), aunque existen estudios que dan cuenta de la participación pública de las mujeres de clase media y alta rioplatense en representación de sus Casas (Aguirrezabala, 2021). Otros agregados domésticos, como las casas de indios, tenían dinámicas familiares muy diferentes, como así también distintos usos de los espacios y los tiempos caracterizados por una mayor relevancia de los lazos de socialibilidad, solidaridad y intercambio en la estructura socioespacial (Zamora, 2010).

Los espacios urbanos como las calles, las plazas y las iglesias eran los lugares en los que se desarrollaba la vida cotidiana y se reunían personas con distinto origen étnico y de clase, como así también eran lugares habitualmente ocupados por mujeres que no pertenecían a las clases sociales altas, dado que, o bien eran esclavas cuyo trabajo se alquilaba o realizaban labores allí para sus dueños, o bien eran libres y vendían productos caseros producidos en el seno de sus hogares para sobrevivir (Zamora, 2010; Fradkin, 2012). Para mantener el orden público existían figuras como la de "escándalo" que permitían a la policía ejercer el control social de las mujeres como un brazo de la fuerza paternal privada matizada, por supuesto, en conformidad con la clase de quienes participaban en las disputas femeninas (Vasallo, 2014; Vaccaroni, 2020). También existía una política sexual sostenida por la administración de justicia como forma de disciplinamiento de las mujeres, especialmente de aquellas que se encontraban solas, y en la que predominaba la adjudicación de la "deshonra" del abuso sexual al marido en lugar de la atención de la víctima como parte del código de conducta y de la sociabilidad colonial (Fradkin, 2012).

Con la Revolución de Mayo y la posterior anexión de territorios pertenecientes a pueblos originarios, las fundación de ciudades persiguió el objetivo de la incorporación de Argentina al mercado mundial (Martínez, 2013), proceso fuertemente marcado tanto por la privatización de ese proceso en favor del patriciado mediante donaciones como por el incentivo a la inmigración para el asentamiento y trabajo en las tierras ocupadas y que requirió, además, de la adecuación de las instituciones jurídicas heredadas del Derecho Indiano para su administración, proceso que no 
estuvo exento de contradicciones (Barcos, 2012). A su vez, con la nefastamente llamada "conquista del desierto" se consolidó, a nivel de subjetivación política, la eliminación del peso específico de la racialidad, política étnica sostenida en el relato nacional hasta hace escasas décadas (Grimson, 2006) ${ }^{2}$.

En este orden de ideas, la regulación de la vida civil tuvo como grandes perdedoras a las mujeres. Comienzan a perder espacios públicos - y asalariados - quedando recluidas en tareas vinculadas a la reproducción social, como el magisterio o al ejercicio de la prostitución en casas de tolerancia (Barrancos, 2007), constituyendo esto último, junto con el manejo de la sanción del abuso sexual por parte de la administración de justicia, en una forma de control social sexuado de los espacios públicos. Hasta la sanción del Código Civil continuaron bajo el designio familiar-masculino, pero se vieron legalmente infantilizadas luego como en el resto de las sociedades occidentales que basaron sus ordenamientos jurídicos en la codificación napoleónica (Barrancos, 2000).

La lucha de las mujeres logró a lo largo de la historia conquistas legales para la mejora de su situación formal frente a los varones pero, no obstante ello, la desigualdad estructural se perpetúa y la producción de las ciudades continúa excluyéndolas. En el siguiente acápite se delinearán los puntos fundamentales de la Teoría de la Reproducción Social a partir de los cuales pueden comenzar a repensarse algunos postulados lefebvrianos.

\section{EL LUGAR DE LA REPRODUCCIÓN SOCIAL EN LAS CIUDADES}

Christian Topalov sostiene que el valor de uso de la ciudad capitalista reside en que es una fuerza productiva dado que concentra las condiciones generales de la producción capitalista (Topalov, 2006 [1979]) y definió a la ciudad, en consecuencia, como "forma de la socialización capitalista de las fuerzas productivas (Topalov, 2006 [1979], p. 9) o "fuerza productiva socializada" (Topalov, 2006 [1979], p. 13), donde la producción y reproducción de la mano de obra se concentran y existen los medios de producción preconstituidos. La ciudad tiene que proveer al capital de las condiciones para la reproducción ampliada de la fuerza de trabajo (Topalov, 2006 [1979]) lo que se traduce en dos espacios separados pero condicionados:

2 Se debe aquí agradecer el diálogo con quienes han participado en la evaluación de este artículo y, acertadamente, han señalado la importancia de la cuestión racial en la configuración de las ciudades latinaomericanas. Si bien por el recorte propio que realiza este texto sería irresponsable por parte de la autora realizar un análisis más exhaustivo, que excede además su propia investigación, queda clara la relevancia de dejar al menos planteada aquí la cuestión desde una mirada histórica del fenómeno. 
puntos de producción de valor y espacios de reproducción de la fuerza de trabajo (Bhattacharya, 2017).

A diferencia de Lefebvre, el feminismo marxista acota la definición de reproducción social a la reproducción de la fuerza de trabajo, lo que permite "hacer foco en el rol del género y la opresión de género en el capitalismo" (Arruzza \& Bhattacharya, 2020, p. 40). La ciudad, desde esta perspectiva, es el escenario donde coinciden la familia, las instituciones públicas que realizan trabajos productivos socializados y las instituciones privadas donde la reproducción social puede convertirse en fuente de ganancias (Arruzza \& Bhattacharya, 2020).

La Teoría de la Reproducción Social, lejos de autoadjudicarse una capacidad explicativa que abarca todas las aristas de la opresión de la mujer tal cual es vivida en el capitalismo, establece las bases socio-materiales para comprender la misma (Ferguson \& McNally, 2013; Varela, 2019; Luparello \& Quiroga, 2019). Inaugurada por Lise Vogel (2013 [1983]), esta teoría vuelve a relacionar explotación y opresión - o, en otras palabras, clase y género - y articula tanto el nivel abstracto como el concreto analizando conceptos teóricos en estrechos lazos con la realidad histórica en la que esas categorías analíticas son aplicadas (Bhattacharya, 2017). Si las mercancías tienen valor de cambio y valor de uso, cuando aparecen bajo apariencia social sólo se manifiesta como valor de uso que, por los flujos de circulación capitalista y a través de lo que Tithi Bhattacharya denomina "an act of "necromancy" ["un acto de 'necromancia"'] (Bhattacharya, 2017, p. 7), es transformado en valor de cambio. La fuerza de trabajo se convierte en una mercancía sin pasar por ese "process of necromancy" (Bhattacharya, 2017, p. 7) como otras mercancías, lo que sugiere que la ontología misma de la fuerza de trabajo trasciende una simple diferenciación entre producción y reproducción (Bhattacharya, 2017).

Esta escuela teórica tiene una profunda raíz espacial con el potencial de complementar las ideas iniciales de Lefebvre y ayudar a su recuperación. En primer lugar, la SRT parte de un entendimiento espacial para desandar el problema de la ontología de la fuerza de trabajo y afirma que existen dos espacios que se encuentran separados aunque mutuamente condicionados: espacios de producción de valor (puntos de producción) y espacios de reproducción de la fuerza de trabajo (Bhattacharya, 2017).

Este enfoque también trasciende la llamada "crisis de los cuidados" - formulación teórica que actualmente ha proliferado en los estudios urbanos con perspectiva de género - en tanto la familia no consigue explicar por sí misma las condiciones, características y grados en los que la clase obrera se produce (Fraser, 2017). Por el contrario, entran aquí en juego las instituciones como la escuela, el sistema de salud, 
los espacios comunitarios de esparcimiento, y los beneficios de la seguridad social (Bhattacharya, 2017), hoy localizadas fundamentalmente en el espacio urbano y sujetos a sus lógicas de producción.

A su vez, permite superar con creces posicionamientos como los del propio Lefebvre en relación a lo doméstico gracias a los aportes que han sabido hacer, por ejemplo, Angela Davis (1981) y Susan Ferguson (2020) al criticar aquel feminismo autoproclamado socialista que exclusivamente se centraba en el trabajo doméstico. En efecto, estas y otras autoras han señalado que la reclusión de las mujeres al espacio doméstico no ha sido una cuestión histórica homogénea, ya que las mujeres atrravesadas por la racialidad y la colonialidad - otras opresiones también abarcadas por la Teoría de la Reproducción Social - históricamente han trabajado como personal doméstico en las casas de las personas blancas (Ferguson, 2020).

Las dimensiones del derecho a la ciudad de las mujeres trabajadoras (Aladro, 2020) pueden ser explicados desde la SRT como el despliegue de opresiones en el nivel de la reproducción de la fuerza de trabajo (Arruzza \& Bhattacharya, 2020). La diferencia en el acceso y goce de los bienes y servicios urbanos en razón de la zona geográfica genera, al final de cuentas, diferentes tipos de trabajadoras porque los procesos de reproducción social se desarrollan de manera diferente. Así, "estos procesos contribuyen a la construcción de diferentes niveles de fuerza de trabajo y también en el mantenimiento de ideas (...) en la sociedad" (Arruzza \& Bhattacharya, 2020, p. 42). Seguidamente se explicará cómo la profundización de la espacialidad que contiene la SRT - específicamente en la noción de "puntos de la reproducción social (Bhattacharya, 2017) - facilita una nueva lectura del espacio social lefebvriano desde el feminismo marxista.

\section{CIUDAD, JERARQUIZACIÓN Y GÉNERO}

Las tendencias hacia las cuales se conduce el espacio abstracto habilitan tanto una explicación de por qué continúan ocurriendo estos parcelamientos como también un punto de ingreso para otra forma de producir conocimiento. El espacio social del capitalismo va hacia la fragmentación, la homogeneización y la jerarquización (Lefebvre, 2013 [1974]). La fragmentación es la división del espacio para su privatización y negociación en unidades como mercancías y la refuerza la propia atomización disciplinar de las ciencias que parcelan y reparten el espacio en función de sus intereses (Butler, 2012). La tendencia a la homogeneidad busca allanar cualquier forma de diversidad que se manifieste en el espacio a una escala global para asegurar la intercambiabilidad de los espacios y los tiempos (Butler, 2012). Por último, la jerarquización es la tendencia que surge de la combinación 
de las dos anteriores y que expone cuál es el rol del Estado en relación al espacio social, pues es su accionar a través de las políticas públicas - principalmente de planificación y de subsidio - que determina el patrón en base al que ese espacio efectivamente se produce (Butler, 2012). Dentro de los objetivos de esas políticas que despliega el Estado, una de las más contundentes es el control social, que se ejerce principalmente mediante la relación centro-periferias (Lefebvre, 1976).

El acceso, la disponibilidad y el goce efectivo de los puntos de reproducción social son partes de las políticas de jerarquización del espacio. El capitalismo moldea el tipo de mano de obra a disposición también a través de la inaccesibilidad de bienes y servicios urbanos vinculados a la reproducción social - escuelas, espacios de ocio, centros de atención de la salud, entre otros - que genera la marginación de los barrios populares, sobre todo en una región tan desigual como América Latina. En este sentido, la urbanización capitalista no sólo condiciona la producción de mercancías sino que también configura "dos procesos de reproducción social diferenciados que objetivan dos tipos de trabajadoras distintas al momento en que éstas llegan a las puertas de capital para vender su fuerza de trabajo" (Arruzza \& Bhattacharya, 2020, p. 41) porque el control social que ejerce el Estado al momento de delimitar el patrón urbano condiciona la reproducción social de las familias proletarias. A continuación se presentan dos ejemplos de procesos diferenciados en función de la disponibilidad de servicios e infraestructuras urbanas que aquí son vistos desde el prisma desde la SRT, si bien sus autoras no plantearon inicialmente sus investigaciones desde tal perspectiva.

En Latinoamérica, Laura Scuro e Iliana Vaca-Trigo (2017) relacionan directamente la zonificación capitalista de las ciudades y las desigualdades espaciales con la mayor carga de tareas vinculadas a la reproducción social - domésticas y de cuidado, de conformidad con el marco teórico de las autoras. Así, recorriendo distintos estudios a lo largo del continente, las autoras sostienen que el acceso deficitario a los servicios e infraestructura urbanos tiene un fuerte impacto negativo en la distribución del tiempo de las mujeres de clase obrera que habitan asentamientos precarios y sobrecarga el tiempo destinado a tareas reproductivas para compensar esa falta.

Otra forma de dar cuenta del impacto del control espacial de la reproducción social es a través de las movilidades que, por supuesto, pueden complementarse con los estudios sobre uso del tiempo, como bien indican Jirón et al (2020). Para Paola Jirón (2017) la movilidad es una red que entrama componentes de producción y reproducción de un número de sujetxs variable y que se relacionan entre sí de diferentes maneras en función de su cotidianidad. La movilidad que resulta de ese 
complejo de relaciones es tipo interdependiente, determina las decisiones respecto de la movilidad y está condicionada por las relaciones sociales de producción capitalista. Las relaciones interdependientes de movilidad son asimétricas y tienen un fuerte sesgo de género - producto de la división sexual del trabajo - , además de estar condicionadas en su constitución y funcionamiento por los recursos económicos de quienes las integran. Así, la priorización de las actividades productivas al momento de establecer los horarios y disponibilidad del servicio público de transporte como la falta de una mirada multiescalar de la movilidad (Jirón, 2017) enfatizan la pauperización de las mujeres de clase trabajadora porque repercuten en la carga de tareas de reproducción social.

\section{CONCLUSIONES}

Haciendo uso de una noción de reproducción social amplia, Henri Lefebvre realizó un aporte renovador a la teoría social marxista al pensar la producción del espacio social rompiendo el paradigma naturalista y estático que caracterizó a esta tradición teórica hasta ese momento, generando un aporte que perdura hasta nuestros días. Simplificando la organización de las mujeres como los movimientos obreros que le fueron contemporáneos (Arruzza, 2013), el sociólogo francés sustentó sus ideas respecto a la "cuestión de la mujer" a partir de dos tesis falsamente adjudicadas a los movimientos feministas, conforme se explicó al dar cuenta de su producción teórica sobre el tema.

El aporte de Silvia Federici respecto de la espacialidad urbana de la acumulación primitiva permite el entendimiento histórico de la expulsión de las mujeres de la esfera pública, su reclusión en el hogar familiar y la degradación de su status legal. Esto puede ser observado también en las ciudades que formaron parte del territorio que actualmente es Argentina que, condicionadas por la colonialidad y la racialidad, desarrollaron sus fuerzas productivas más tardíamente, pero mantuvieron las estrategias de subordinación de las mujeres dictadas en los países del viejo continente y generaron otras prácticas espaciales específicas, como las signadas por la esclavitud.

Por otro lado, las contribuciones de la teoría unitaria permiten comprender la contemporaneidad del lugar de las mujeres trabajadoras en las metrópolis e identificar las consecuencias del acceso diferencial de las mujeres a la ciudad y los bienes urbanos en función de las necesidades del capital. En este sentido, no sólo deben ser atendidas las características de los puntos de reproducción social urbanos sino la vida cotidiana de las mujeres, para identificar también cómo la 
falta de acceso a los servicios urbanos de reproducción social las pauperizan en tanto parte del proletariado y cómo interviene el Estado mediatizando esa situación.

Las estrategias de apropiación de la ciudad por las que abogaba Lefebvre (2013 [1974]) para la recuperación del valor de uso se relacionan, sobre todo, a la resistencia autogestiva para desafiar y controlar el espacio abstracto "towards the progressive democratisation of institutions and decision making processes within workplaces, representative structures, cities and regions" (Butler, 2012, p. 100). Históricamente, la academia, poniendo en el eje la producción de valor, ha relacionado esta idea con la formación de barrios populares como las favelas en tanto reapropiación del valor de uso y la creatividad para combatir al mercado y la dominación (Butler, 2012). Sin embargo, "pretender entender el sistema capitalista en su conjunto mirando sólo cómo se extrae el plusvalor, es como querer entender al ser humano mirando cómo late su corazón” (Arruzza \& Bhattacharya, 2020, p. 45). En el caso de las ciudades, suponer que puede comprenderse el espacio urbano como totalidad prestando atención exclusivamente a su valor de cambio, equivale al mismo error. A la par de la formación de barrios populares como combate a la mercantilización inmobiliaria, las mujeres socializan las funciones de reproducción social frente a la inaccesibilidad de servicios como la alimentación, la salud, la educación, el transporte, y la lista sigue. La potencialidad de la SRT para complementar la teoría lefebvriana radica en que brinda la posibilidad de explicar las ciudades integrando explotación y opresión mientras se recuperan las experiencias de las mujeres trabajadoras que generan permanentemente estrategias de supervivencia colectivas en un espacio que, desde la acumulación primitiva, las expulsó. En este sentido, a pesar de que los ejemplos traídos en este artículo abordan específicamente el acceso a servicios formales de reproducción social externos al hogar, la SRT también es una llave de ingreso a las estrategias de reproducción social interfamiliares, asalariadas y incluso comunitarias (Ferguson, 2020).

\section{REFERÊNCIAS BIBLIOGRÁFICAS}

AGUIRREZABALA, Marcela. (2021). "En defensa de la Casa: Mujeres y política entre la ruptura del orden colonial y la independencia”. En: Tejerina, M. V.; Cantera, C. S. (eds.), Implicar al otro. Espacio, territorio y poder entre la colonia y las Provincias Unidas del Río de la Plata. Bahía Blanca: EDIUNS, p. 11-44.

ALADRO, Almendra (2020). "El acceso al derecho a la ciudad de las mujeres”. II Coloquio Internacional de Conflictos Urbanos. Jerez de la Frontera: UCA.

ARRUZZA, Cinzia (2013). Dangerous Liaisons: The marriages and divorces of Marxism and Feminism.Exeter: Resistance Books. 
ARRUZZA, Cinzia; BHATTACHARYA, Tithi (2020). “Teoría de la Reproducción social: Elementos fundamentales para un feminismo marxista”. Archivos de historia del movimiento obrero y de la izquierda, v.8, n. 16, p. 37-69.

BARCOS, María Fernanda (2012). "El influjo del Derecho Indiano en la legislación sobre ejidos de la Provincia de Buenos Aires, 1782-1870". Revista de Indias, v. 72, n. 256, p. 687-716.

BARRANCOS, Dora. (1994). "Entre la celebración y el escarnio: mujeres contestatarias (1890-1900)". In: FLETCHER, L. (Org.). Mujeres y culturas en la Argentina del siglo XIX. Buenos Aires: Feminaria.

BARRANCOS, Dora (2000). "Inferioridad jurídica y encierro doméstico". In: GIL LOZANO, F.; PITA, V.; INI, M. G. (orgs.). Historia de las Mujeres en la Argentina. Tomo I. Buenos Aires: Taurus.

BARRANCOS, Dora (2007).Mujeres en la sociedad argentina: Una historia de cinco siglos. Sudamericana: Buenos Aires.

BHATTACHARYA, Tithi (2017). "Introduction: Mapping Social Reproduction Theory. In: BHATTACHARYA, Tithi (ed.).Social Reproduction Theory: Remapping Class, Recentering Oppression. London: Pluto Press, p. 1-20.

BUTLER, Chris (2012). Spatial politics, Everyday Life and the Right to the City. Nueva York: Routledge.

CANESTRARO, María Laura (2016). "Sobre el derecho a la ciudad y el acceso al suelo urbano: Reflexiones a partir de intervenciones estatales recientes (Mar del Plata, 2012-2015)”. Estudios Socioterritoriales. Revista de Geografía, n. 20, p. 57-74.

DAVIS, Angela (1981). Women, Race, and Class. New York: Vintage Books.

FALÚ, Ana (2014). "El derecho de las mujeres a la ciudad: Espacios públicos sin discriminaciones y violencias". Revista Vivienda y Ciudad, v. 1, p. 10-28.

FALÚ, Ana (2016). "La omisión de género en el pensamiento de las ciudades”. In: BORJA, Jordi; CARRIÓN, Fernando; CORTI, Marcelo (eds.). Ciudades para cambiar la vida: Una respuesta Hábitat III. Buenos Aires: Café de las Ciudades, p. 159-179.

FAVELUKES, G. (2011). Figuras y paradigmas. Las formas de Buenos Aires (1740-1870). Anales del Instituto de Arte Americano e Investigaciones Estéticas "Mario J. Buschiazzo", v. 41, n. 1.

FEDERICI, Silvia (2010). Calibán y la bruja. Madrid: Traficantes de Sueños.

FEDERICI, Silvia (2018). El patriarcado del salario: Críticas feministas al marxismo. Madrid: Traficantes de Sueños.

FERGUSON, Susan (2020). Women and Work: Feminism, Labour and Social Reproduction. London: Pluto Press. 
FERGUSON, Susan; MC NALLY, David (2013). "Capital, Labour Power and GenderRelations: Introduction to the Historical Materialism Edition of Marxism and the Oppression of Women". In: VOGEL, Lise. Marxism and the Oppression of Women: Towards a Unitary Theory. Leiden: Brill, p. XVII-XL.

FRADKIN, Raúl (2012). Historia de la provincia de Buenos Aires. Gonnet: EDHASAUNIPE.

FRASER, Nancy (2017). "Crisis of Care? On the Social Reproductive Contradictions of Contemporary Capitalism”. In: BHATTACHARYA, Tithi (ed.). Social Reproduction Theory: Remapping Class, Recentering Oppression. London: Pluto Press, p. 21-36. GRIMSON, Alehandro (2006). "Nuevas xenofobias, nuevas políticas étnicas en Argentina”. In: GRIMSON, A.; JELIN, E. Migraciones regionales hacia la Argentina: Diferencia, desigualdad y derechos. Buenos Aires: Prometeo, p. 69-97.

JIRÓN, Paola (2017). "Planificación urbana y de trasporte a partir de relaciones de interdependencia y movilidad del cuidado”. In: NIEVES RICO, María; SEGOVIA, Olga (eds.). ¿Quién cuida en la ciudad? Aportes para políticas urbanas de igualdad. Santiago de Chile: CEPAL, p. 405-431.

JIRÓN, Paola, CARRASCO, Juan-Antonio; REBOLLEDO, Marcela (2020). “Observing gendered interdependent mobility barriers using an ethnographic and time use approach". In: Transportation Research, n. 140, p. 204-214.

LEFEBVRE, Henri (1961). Introducción al marxismo. Buenos Aires: EUDEBA.

LEFEBVRE, Henri. (1976). Tiempos equívocos. Barcelona: Kairós.

LEFEBVRE, Henri (1978). De lo rural a lo urbano. Barcelona: Cultura Libre.

LEFEBVRE, Henri (2013 [1974]). La producción del espacio. Madrid: Capitán Swing Libros. LEFEBVRE, Henri (2017 [1968]). El derecho a la ciudad. Madrid: Capitán Swing Libros. LUPARELLO, Velia; QUIROGA, Manuel (2019). "Sobre las relaciones entre feminismo y marxismo: un aporte desde la Teoría de la Reproducción Social”. In: CÓRDOBA, Liliana; LA SERNA, Carlos; CRISTINI, Romina (orgs.). I Congreso Nacional de Ciencias Sociales: Las ciencias sociales a 100 años de la Reforma Universitaria, Córdoba, p. 432-441.

MARTÍNEZ, Mónica (2013). "Los pueblos del Desierto: Conquista, urbanización y colonización del territorio de La Pampa, Argentina (1879-1930)”. In: Registros. Revista de Investigación Histórica, n. 10, p. 38-59.

SCURO, Laura; VACA-TRIGO, Iliana (2017). "La distribución del tiempo en el análisis de las desigualdades de las ciudades de América Latina”. In: NIEVES RICO, María; SEGOVIA, Olga (eds.). ¿Quién cuida en la ciudad?Aportes para políticas urbanas de igualdad. Santiago de Chile: CEPAL, p. 117-148. 
SKEGGS, Beverly (2019). Mujeres respetables: Clase y género en los sectores populares. Los Polvorines: Universidad Nacional de General Sarmiento.

SMITH, Doroty (2005). Institutional ethnography: A sociology for people. Toronto: Rowman Altamira.

VACCARONI, Agustina (2020). "La policía y el proceso de territorialización de la ciudad de Buenos Aires: Medio siglo de proyectos, 1782-1824". Quinto Sol, v. 24, n. 1, p. 1-22.

VARELA, Paula (2019). “iExiste un feminismo socialista en la actualidad? Apuntes sobre el movimiento de mujeres, la clase trabajadora y el marxismo hoy". Revista THEOMAI, n. 39, p. 4-20.

VASALLO, Jaqueline (2014). "Judicializar el escándalo: disputas femeninas y control social. Cabildo de Córdoba de Tucumán, 1782-2010”. Mouseion, n. 18, p. 115-131.

VOGEL, Lise (2013 [1983]). Marxism and the Oppression of Women: Towards a Unitary Theory. Leiden: Brill.

ZAMORA, Romina (2010). "Lo doméstico y lo público. Los espacios de sociabilidad de la ciudad de San Miguel de Tucumán a fines del siglo XVIII y comienzos del XIX”. Nuevo Mundo, Nuevos Mundos, n. 10. 